\title{
Synergistic Antibacterial Activity of Combined Antimicrobials and the Clinical Outcome of Patients With Carbapenemase-Producing Acinetobacter baumannii Infection
}

OPEN ACCESS

Edited by:

Constantinos Tsioutis, European University Cyprus, Cyprus

Reviewed by:

Jozsef Soki,

University of Szeged, Hungary

Petros loannou,

University Hospital of Heraklion,

Greece

*Correspondence:

Xiaoju LV

Ivxj33966@126.com

Specialty section:

This article was submitted to Antimicrobials, Resistance

and Chemotherapy,

a section of the journal

Frontiers in Microbiology

Received: 09 March 2020

Accepted: 19 August 2020

Published: 15 October 2020

Citation:

Qu J, Yu R, Wang Q, Feng $C$ and Lv X (2020) Synergistic Antibacterial Activity of Combined Antimicrobials and the Clinical Outcome of Patients

With Carbapenemase-Producing

Acinetobacter baumannii Infection.

Front. Microbiol. 11:541423.

doi: 10.3389/fmicb.2020.541423

\author{
Junyan Qu ${ }^{1}$, Rujia Yu ${ }^{1}$, Qujue Wang ${ }^{2}$, Chunlu Feng ${ }^{1}$ and Xiaoju Lv ${ }^{1 *}$ \\ ${ }^{1}$ Center of Infectious Diseases, West China Hospital, Sichuan University, Chengdu, China, ${ }^{2}$ Department of Infectious \\ Diseases, Renshou County People's Hospital, Renshou, China
}

This study aimed to explore the activity of combined antimicrobials in vitro, and the relationship among resistance mechanisms, antimicrobial regimens, and the clinical outcome of patients with carbapenem-resistant Acinetobacter baumannii (CRAB) infections in western China. A total of 89 CRAB strains were collected from patients with CRAB infection from January 2018 to June 2018. The checkerboard assay was used to study the combined effects in vitro. Carbapenemase-encoding genes were detected by polymerase chain reaction (PCR) or multiplex PCR technique. The clinical data of 86 patients were collected. CRAB showed high susceptibility to tigecycline $(91.01 \%$ inhibition) and polymyxin (83.15\% inhibition). Polymyxin plus sulbactam exhibited the highest synergistic effect at a rate of $82.35 \%$. Production of carbapenemase (blaOXA-23) was the main resistance mechanism of CRAB to carbapenem (95.35\%). Excessive expression of active efflux pump genes ( $a d e B$, adeJ, and abeM) and deletion of the CarO protein accounted for $13.95 \%$ (12/86) and $84.88 \%$ (73/86), respectively. The synergistic effect of the sulbactam-based combination was higher than that of the polymyxin B-tigecycline combination for carbapenemase-producing CRAB $(P<0.05)$. The clinical outcome was not affected by the resistance mechanisms $(P>0.05)$. Advanced age, multiple organ dysfunction syndromes (MODS), and admission to the intensive care unit (ICU) were associated with treatment failure $(P<0.05)$. Appropriate antibiotic therapy did not improve the clinical outcome of critically ill patients. Higher minimum inhibitory concentrations (MICs) of tigecycline were associated with treatment failure $(P<0.05)$. A multivariate analysis showed that ICU stay $(\mathrm{OR}=15.123,95 \% \mathrm{Cl}: 2.600-87.951$, $P=0.002)$ and procalcitonin $\geq 2 \mathrm{ng} / \mathrm{ml}(\mathrm{OR}=2.636,95 \% \mathrm{Cl}: 1.173-5.924, P=0.019)$ were the risk factors for treatment failure. In conclusion, this study demonstrated that the sulbactam-based combination exhibited a synergistic effect in vitro. The clinical outcome of patients was not associated with resistance mechanisms. This indicates that the early control of the progression from infection to severe disease may be important.

Keywords: carbapenem-resistant Acinetobacter baumannii, infection, resistance mechanism, synergistic effect, clinical outcome 


\section{INTRODUCTION}

Carbapenem-resistant Acinetobacter baumannii (CRAB) is at the top of the World Health Organization's list of drugresistant bacteria and poses a significant threat to human health (Willyard, 2017). It can cause severe infections including pneumonia, bloodstream infection, abdominal infection, and skin and soft tissue infection, for which almost no treatment is available (Willyard, 2017). Data from the China Antimicrobial Surveillance Network (CHINET) has shown that the percentage of imipenem-resistant Acinetobacter baumannii (AB) increased from $39.0 \%$ in 2005 to $78.1 \%$ in 2018 (CHINET, 2019). Infections caused by CRAB have high mortality due to limited antimicrobial options, and CRAB bloodstream infection tends to occur in critically ill patients along with rapid disease progression and higher mortality. A previous study showed that the 30-day mortality of patients with CRAB bacteremia in the intensive care unit (ICU) was as high as $79.8 \%$ (Kim et al., 2012). The new $\beta$-lactamase inhibitor ceftazidime/avibactam presents an opportunity to improve the survival of patients with carbapenem-resistant Pseudomonas aeruginosa and Enterobacteriaceae infections, but the susceptibility of CRAB to ceftazidime/avibactam was poor (Hsueh et al., 2019). Several new antimicrobial agents, including aztreonam/avibactam, cefiderocol, and eravacycline, might be promising therapeutic options in treating $\mathrm{CRAB}$ infections (Leone et al., 2019). Currently, the treatment options for CRAB infections are limited, including colistin, tigecycline, sulbactam, and aminoglycosides (Karaiskos et al., 2017). Therefore, knowing which of the existing antimicrobials to select in controlling CRAB infections is a huge challenge for clinicians. Observational studies have shown no difference in statistics between monotherapy and combination therapy in mortality and clinical efficacy in patients with CRAB infections (Karaiskos et al., 2017). There are also no convincing studies that recommend the combination of carbapenems, colistin, or sulbactam (Karaiskos et al., 2017).

The resistance mechanisms of CRAB to carbapenem mainly include the production of carbapenemases (especially class $\mathrm{D}$ carbapenemases) in $\mathrm{AB}$, excessive expression of an active efflux pump, functional loss of outer-membrane porins (Brink, 2019). Different antimicrobial agents have different antimicrobial mechanisms and targets. Thus, understanding the antibiotic resistance mechanisms exhibited by CRAB will help clinicians to choose the antimicrobial regimens appropriately. At present, some studies have focused on the resistance mechanisms of clinical strains (Jiang et al., 2019; Li S. et al., 2019), and other studies have focused on observational research of the clinical efficacy of different antimicrobial regimens in the treatment of CRAB infections (Elsayed et al., 2019; Russo et al., 2019). Few clinical studies have compared the efficacy of the existing antimicrobial regimens based on different resistance mechanisms. Therefore, it is extremely important to know how to better select from the existing antimicrobials and optimize the treatment regimen according to the antibiotic resistance mechanisms to achieve a better clinical outcome.

This study explored the following three aspects: (1) The in vitro activities of imipenem (IPM), biapenem (BIP), sulbactam
(SUL), tigecycline (TGC), or polymyxin $\mathrm{B}(\mathrm{PB})$, and the combination of IPM-TGC, IPM-BIP, PB-TGC, TGC-SUL, PBSUL against CRAB were determined. (2) It investigated the mechanisms of carbapenem resistance in clinical CRAB strains. (3) we evaluated the relationship between resistance mechanisms, antimicrobial regimens, and clinical outcomes in patients with CRAB infections.

\section{MATERIALS AND METHODS}

\section{Bacterial Strains and Drugs}

For this study, eighty-nine non-repeated clinical CRAB strains were collected from patients with CRAB infection between January 2018 and June 2018 in West China Hospital, Sichuan University (a 4300-bed academic tertiary-care hospital). We conducted identification and antimicrobial susceptibility tests (AST) on the VITEK-2 COMPACT automated microbiology system (BioMerieux, France). Species identification was confirmed by detecting and sequencing the $g y r B$ and $r p o B$ genes. The following drugs were used in these experiments: imipenem/cilastatin sodium (Merck Sharp \& Dohme Corp., New Jersey, United States), biapenem (Chia Tai Tianqing Pharmaceutical Co., Ltd., Jiangsu, China), cefoperazone/sulbactam sodium (Pfizer, New York, United States), polymyxin B (SPH NO.1 Biochemical \& Pharmaceutical Co., Ltd., Shanghai, China), tigecycline (Wyeth Pharmaceutical Co., Ltd., Carolina, United States), and carbonyl cyano-p-chlorphenizone (CCCP) (Sigma-Aldrich, Inc., United States).

\section{Clinical Isolates and Antimicrobial Susceptibility Testing, and Synergistic Testing and Determination of Efflux Pump Activity}

The minimum inhibitory concentrations (MICs) of IPM, BIP, TGC, SUL, and PB were determined using the broth microdilution method following CLSI guidelines (CLSI, 2017). The susceptibility breakpoints of the antimicrobials used against A. baumannii in this study were in accordance with the CLSI criterion (CLSI, 2017). The breakpoint for BIP was defined as $\geq 8 \mathrm{mg} / \mathrm{L}$ resistant strains based on the breakpoints for other carbapenems against A. baumannii, because of the lack of CLSI, European Committee on Antimicrobial Susceptibility Testing (EUCAST), or FDA-approved breakpoint for BIP. The breakpoints for TGC were based on the FDA-approved tigecycline breakpoints for the Enterobacteriaceae, $\leq 2 \mathrm{mg} / \mathrm{L}$ susceptible and $\geq 8 \mathrm{mg} / \mathrm{L}$ resistant. Escherichia coli ATCC 25922 was used as the reference strain. The synergistic effects of antimicrobial combinations were determined using the checkerboard method with Mueller-Hinton broth (Gordon et al., 2010). The fractional inhibitory concentration indices (FICI) were calculated for each of the paired antimicrobials using the following equation: the MIC of drug A in combination/the MIC of drug A alone + the MIC of drug B in combination/the MIC of drug $\mathrm{B}$ alone. $\mathrm{FICI}<1$, FICI $=1.0$, and $1<\mathrm{FICI}<2.0$ were used to 
define synergy, addition, and indifference, respectively, according to a previous study (Yoon et al., 2004). MICs of IPM were observed in the absence and the presence of CCCP $(10 \mu \mathrm{g} / \mathrm{mL})$. A significant inhibition effect was defined as a four-fold or more reduction in IPM MICs in the presence of CCCP. Efflux pump activity was determined for the efflux pump positive isolates.

\section{Polymerase Chain Reaction (PCR) and Nucleotide Sequencing}

Classes A and D carbapenemase-encoding genes ( $b l a_{\mathrm{GES}}, b l a_{\mathrm{KPC}}$, $b l a_{\mathrm{OXA}-51}, b l a_{\mathrm{OXA}-23}, b l a_{\mathrm{OXA}-24}, b l a_{\mathrm{OXA}-58}$, and $\left.b l a_{\mathrm{OXA}-143}\right)$ and class B metallo- $\beta$-lactamase genes (bla $a_{\mathrm{IMP}}, b l a_{\mathrm{VIM}}, b l a_{\mathrm{SPM}}$, $b l a_{\mathrm{GIM}}, b l a_{\mathrm{SIM}}$, and $\left.b l a_{\mathrm{NDM}}\right)$ were detected by the PCR method or multiplex PCR technique as previously described (Poirel et al., 2000; Woodford et al., 2006; Ellington et al., 2007; Higgins et al., 2010). The primers were synthesized and the amplicons obtained were sequenced by the Beijing Genomics Institute. The sequences were analyzed with the NCBI BLAST program ${ }^{1}$.

\section{Fluorescence Quantitative Real-Time RT-PCR}

The expression levels of efflux system genes (adeB, ade), and $a b e M)$ were assessed using fluorescence quantitative realtime reverse transcription-PCR (qRT-PCR). The adeB gene was screened as described previously (Peleg et al., 2007) and new primers were designed for the adeJ (adeJ-F: ATGAGAAA CTGATTGCAGCTC; adeJ-R: TGAGGAGTATCTTCCTGAC CA) and abeM (abeM-F: AGGCTTCGGCTTATCGAAAC; abeM-R: AGAGGGCTAAGGACCAATGC) genes.

The RNA was extracted using TaKaRa RNAiso Plus (TaKaRa Bio Inc., Japan), and cDNA was synthesized using the PrimeScript RT reagent Kit with gDNA Eraser (TaKaRa Bio Inc., Japan). Real-time PCR assays were performed using an Option 2 real-time PCR detection system (Bio-Rad Inc., United States) with the SsoFast EvaGreen Supermix Kit (BioRad Inc., United States). The 16S rRNA gene was used as the internal control gene. Expression analysis was performed using the $2^{-\Delta \Delta C t}$ method (Livak and Schmittgen, 2001) to compare the relative expression of the mRNA with that of A. baumannii ATCC 19606.

\section{Western Blot Analysis}

Western blot analysis was used to evaluate the level of the outer membrane protein CarO expression for each isolate. Bacterial cells were mixed with radioimmunoprecipitation (RIPA) buffer (3 ml RIPA buffer/g). After centrifugation, the supernatant was collected. Western blotting was performed as previously described (Goh et al., 2013). The CarO-specific antibody was generated in a rabbit by Abmart Biomedicine Co., Ltd. (Shanghai, China; 1: 1000 dilution). The secondary antibody was horseradish peroxidase (HRP) conjugated goat anti-rabbit IgG (Fumaisi Biotechnology Co., Ltd., Nanjing, China; 1: 2000 dilution).

\footnotetext{
${ }^{1}$ www.ncbi.nlm.nih.gov/blast/
}

\section{Clinical Data}

Detailed clinical information of patients with CRAB infection, including demographic characteristics, laboratory data, antibiotic treatment regimens, and clinical outcomes, were collected from their medical records. The clinical and microbiological diagnosis of CRAB infection was defined according to the criteria of the Centers for Disease Control and Prevention (CDC) (Horan et al., 2008). Colonization was excluded before the beginning of this study. CRAB was defined as A. baumannii demonstrating resistance or intermediate susceptibility to one or more of meropenem or IPM, according to the AST results. Appropriate antibiotic treatment was defined as the use of at least one active drug against $\mathrm{CRAB}$ within 5 days of the date of sampling. Treatment outcomes were evaluated at 28 days after clinical samples were taken from patients. Clinical outcomes were defined as treatment success or treatment failure (death or disease progression). This study was approved by the Ethics Committee of West China Hospital, Sichuan University.

\section{Statistical Analysis}

Statistical analyses were performed using SPSS version 22.0 for Windows (SPSS Inc., Chicago, United States). Normality tests for quantitative variables were assessed by the ShapiroWilk test. Significance testing was performed using the Student's $t$ test or Mann-Whitney $\mathrm{U}$ test. Categorical variables were compared using the Chi-square test or Fisher's exact test. Risk factors for 28-day treatment outcomes of patients with CRAB infection were identified using a logistic regression model. All variables with $P<0.05$ in univariate analysis were entered into the multivariate analysis by using the forward conditional method. $P<0.05$ (two-tailed) was considered statistically significant.

\section{RESULTS}

\section{In vitro Antibacterial Activity of Antimicrobials Alone and in Combination}

The sensitivity rates of CRAB to IPM, BIP, SUL, TGC, and colistin were $0,0,2.25 \%$ (2/89), 91.01\% (81/89), and 83.15\% (74/89), respectively. Some strains were selected to evaluate the drug interaction in antibiotic combinations according to the MIC values (excluding highly susceptible and resistant strains). MICs of antimicrobials against CRAB alone and in combination are shown in Table 1. For all drug combinations, a synergistic effect was the most common interaction. PB-SUL showed the highest synergistic effect at a rate of $82.35 \%$ (14/17), followed by TGC-SUL at a rate of $73.91 \%(17 / 23)$.

\section{Association of the Clinical Outcome and Resistance Mechanisms}

We collected the clinical data of 86 patients with CRAB infections. Three patients were lost because, by the time the diagnosis was made, they were transferred to other hospitals. The distribution of carbapenem resistance mechanisms in these 


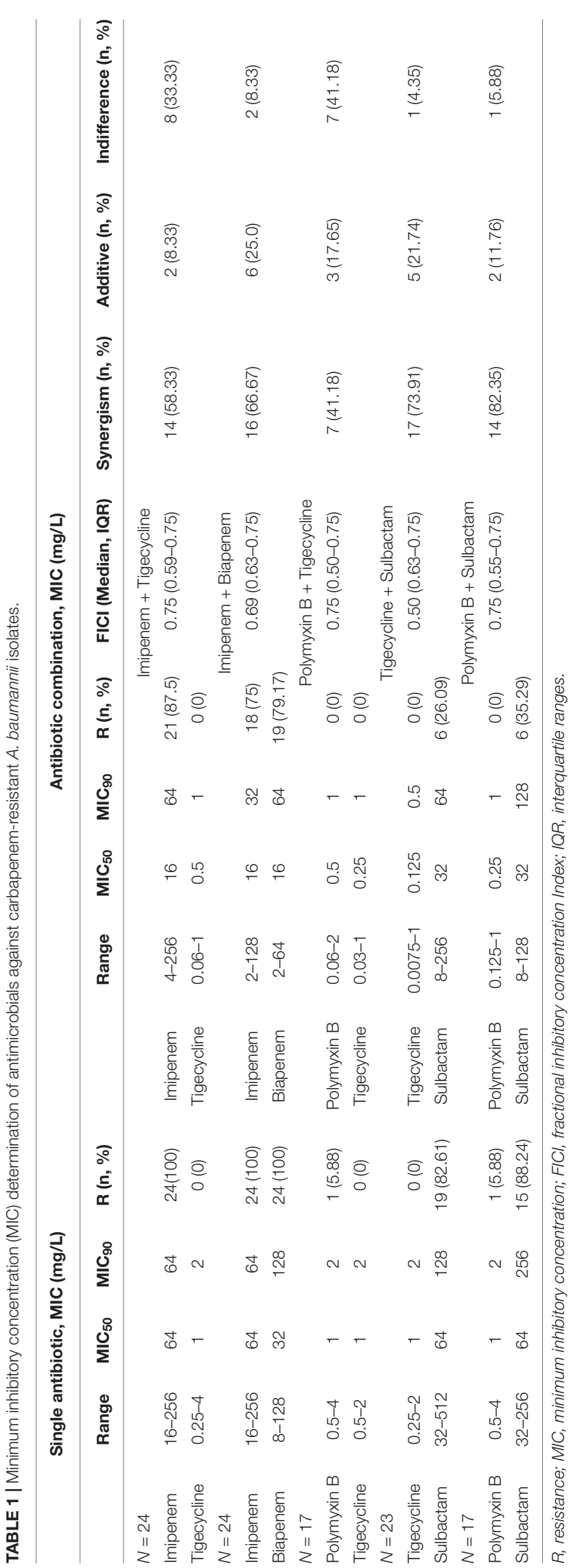

clinical isolates is shown in Table 2 . The only detectable carbapenemase gene was bla OXA-23 $_{2}(82,95.35 \%)$. All strains detected the naturally occurring bla $a_{\mathrm{OXA}-51}$ gene $(86,100.00 \%)$. The sequences of OXA51 and OXA23 obtained in this study have been submitted to GenBank and the accession numbers are MT757793 and MT757794, respectively. Other carbapenemaseencoding genes were not detected. The MIC values of 12 isolates were decreased at least four-fold in the presence of CCCP, and they were also the active efflux phenotype positive strains. The relative expression of $a d e B$, adeJ, and $a b e M$ had increased by approximately 4.4-fold, 4.1-fold, and 3.8-fold, respectively, in $\mathrm{CRAB}$ isolates than in the sensitive strain. The positive rate of CarO porin in the treatment success group was higher than that in the treatment failure group $(P<0.05)$.

\section{The in vitro Antibacterial Activity of Antimicrobial Combination in CRAB With Different Resistance Mechanisms}

The synergistic effect of PB in combination with SUL was higher than that of the other drug combinations against CRAB strains $(P<0.05)$. In the strains with carbapenemases, the synergistic effect of the SUB-based combination was higher than that of PBTGC combination $(P<0.05)$. For other resistance mechanisms, no drug combination showed a statistically significant difference in the synergistic effect $(P>0.05)$ (see Table 3 ).

\section{Clinical Characteristics of Patients With CRAB Infection}

After excluding 4 patients with non-carbapenemase-producing CRAB infection, a total of 82 patients with CRAB infections (mean age $58.05 \pm 17.54$ years; 51 male) were included in this study (Table 4$)$. The treatment failure rate was $28.05 \%(23 / 82)$ within 28 days of the specimens being taken. Patients at an advanced age, those with multiple organ dysfunction syndromes (MODS), and those admitted in the ICU had a higher rate of treatment failure $(P<0.05)$. Patients in the treatment failure group had higher levels of procalcitonin (PCT) and percentage of neutrophils, and a lower level of estimated glomerular filtration rate (eGFR) than those in the treatment success group $(P<0.05)$. The treatment failure group had a higher proportion of CRAB patients with bloodstream infections than the treatment success group $(P<0.05)$. More patients had a poor clinical outcome even if they were given appropriate antibiotic therapy $(P<0.05)$. Patients infected with CRAB strains with lower MICs of TGC had a better clinical outcome $(P=0.021)$.

\section{Risk Factors for a Poor Treatment Outcome of Patients With CRAB Infection}

Multivariate analysis showed that ICU stay (OR $=15.123,95 \%$ CI: 2.600-87.951, $P=0.002)$ and PCT $\geq 2 \mathrm{ng} / \mathrm{ml}(\mathrm{OR}=2.636$, 95\% CI: $1.173-5.924, P=0.019)$ were independent risk factors for treatment failure in patients with $\mathrm{CRAB}$ infection, as shown in Table 5. 
TABLE 2 | Distribution of carbapenem resistance mechanisms in clinical isolates of carbapenem-resistant Acinetobacter baumannii.

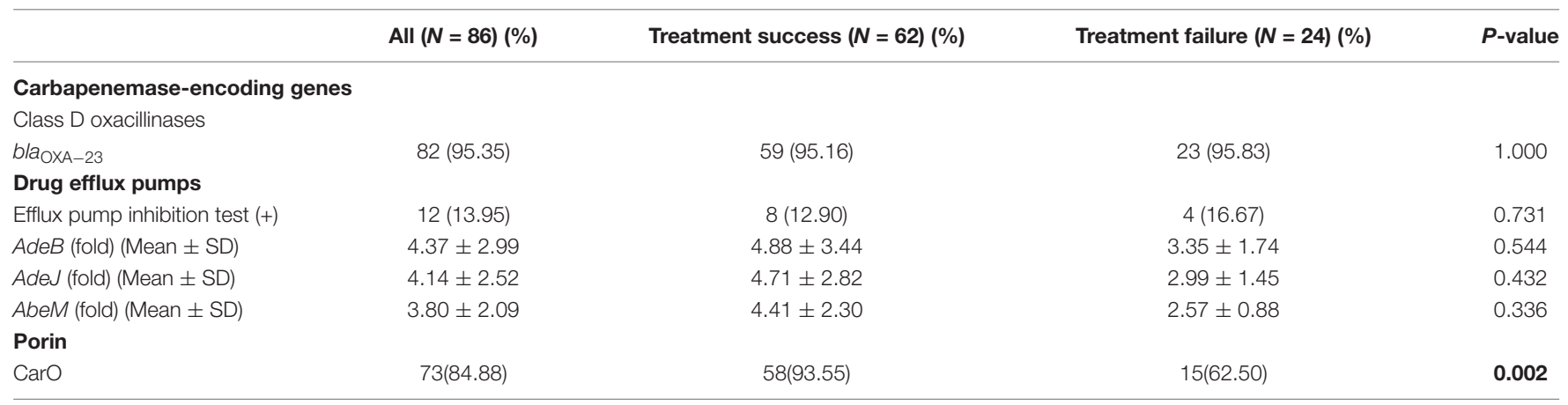

SD, standard deviation. These bold values emphasize that the difference is statistically significant.

TABLE 3 | The synergistic effect of drug combinations in carbapenem-resistant Acinetobacter baumannii with different resistance mechanisms.

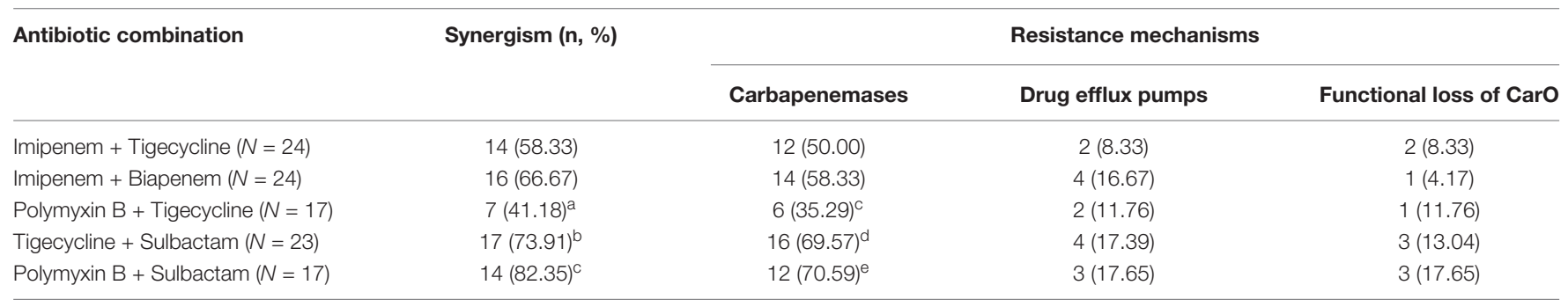

$p^{a b}<0.05 ; P^{a c}<0.05 ; P^{c d}<0.05 ; P^{c e}<0.05$

TABLE 4 | Clinical characteristics in patients with carbapenem-resistant Acinetobacter baumannii infection.

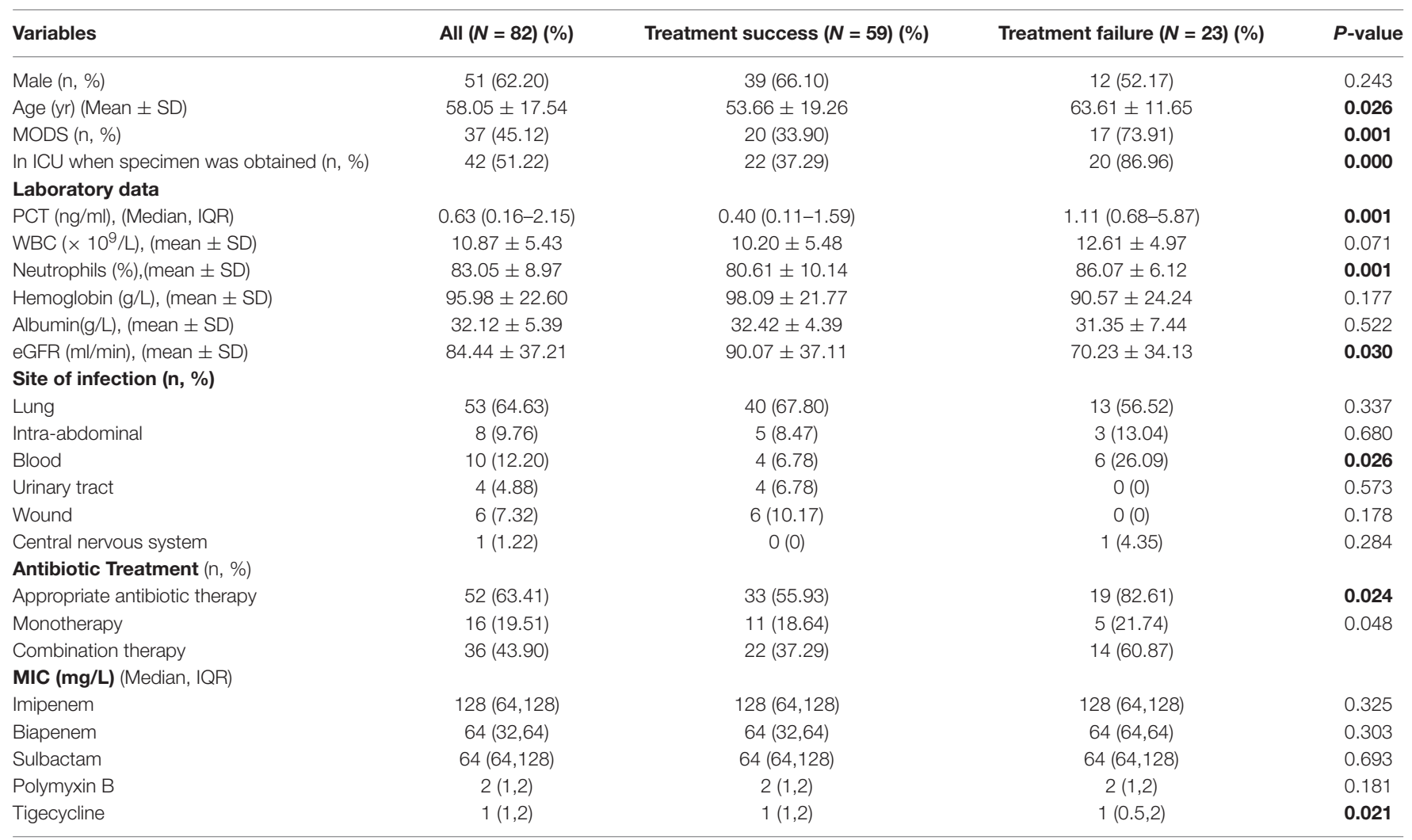

MODS, multiple organ dysfunction syndromes; ICU, intensive care unit; PCT, procalcitonin; WBC, white blood cell; eGFR, estimated glomerular filtration rate; MIC, minimum inhibitory concentration; SD, standard deviation; IQR, interquartile ranges. These bold values emphasize that the difference is statistically significant. 
TABLE 5 | Risk factors associated with treatment failure of patients with carbapenem-resistant Acinetobacter baumannii infection.

\begin{tabular}{lccc}
\hline Variables & P-value & Odds ratio(OR) & 95\% CI \\
\hline Ages & 0.156 & 1.037 & $0.986-1.089$ \\
MODS & 0.114 & 3.752 & $0.729-19.320$ \\
In ICU when specimen was obtained & $\mathbf{0 . 0 0 2}$ & 15.123 & $2.600-87.951$ \\
PCT ( $\geq 2$ ng/ml) & $\mathbf{0 . 0 1 9}$ & 2.636 & $1.173-5.924$ \\
Neutrophils (Percent) & 0.950 & 1.034 & $0.359-2.982$ \\
eGFR & 0.660 & 1.163 & $0.593-2.280$ \\
Appropriate antibiotic therapy & 0.798 & 1.248 & $0.227-6.856$ \\
MICs of tigecycline & 0.799 & 0.870 & $0.296-2.551$ \\
Bloodstream infection & 0.975 & 1.030 & $0.166-6.406$
\end{tabular}

MODS, multiple organ dysfunction syndromes; ICU, intensive care unit; PCT, procalcitonin; eGFR, estimated glomerular filtration rate; MIC, minimum inhibitory concentration. These bold values emphasize that the difference is statistically significant.

\section{DISCUSSION}

In this study, we found that $\mathrm{CRAB}$ displayed high sensitivity to TGC and $\mathrm{PB}$, which is similar to that reported in many previous reports (He et al., 2015; Yazdansetad et al., 2019). Despite their in vitro activity against CRAB, the results of studies on the clinical effectiveness of TGC have been mixed, and even show a higher mortality risk (McGovern et al., 2013). A low serum concentration of TGC is inadequate to eradicate CRAB, and this limits its use in $\mathrm{CRAB}$ bloodstream infection. The role of TGC in treating $\mathrm{CRAB}$ infections remains controversial. Nephrotoxicity and neurotoxicity of colistin limited its clinical use, and patients with carbapenem-resistant bacterial infections receiving colistin monotherapy had a less than optimal outcome (Zusman et al., 2017). The combination of several antimicrobial agents produced synergistic effects in this study, especially the PB-SUL combination. Although many previous studies have demonstrated the synergistic or additive effects of an antimicrobial combination (He et al., 2015; Le Minh et al., 2015), the antibacterial activity in vitro does not necessarily reflect activity in vivo. In clinical practice, the efficacy of TGC and polymyxin was uncertain even when used in combination with other antimicrobials (Zusman et al., 2017; Doi, 2019). A previous study showed that the polymyxin-vancomycin combination produced a synergistic effect in vitro (Bae et al., 2016). Vancomycin may enhance the permeability of polymyxin on the A. baumannii outer membrane, and perhaps other antimicrobial agents, such as ceftazidime, aztreonam, and meropenem, may also have this effect, which can be studied further.

A high percentage of OXA-23-positive isolates were observed in this study. Carbapenemase production was the main resistance mechanism of CRAB to carbapenem. However, different types of resistance mechanisms in CRAB did not affect the clinical outcome. A previous study found that patients infected with

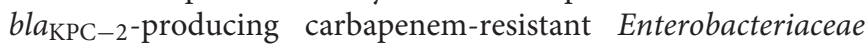
(CRE) had poor outcomes (Wang et al., 2018). Mutations in bla $a_{\mathrm{KPC}-3}$ in CRE may lead to a decrease in the antibacterial activity of ceftazidime-avibactam (Haidar et al., 2017). Early detection of the carbapenemase type might help improve the prognosis of patients with CRE infections. With respect to CRAB, studies on the relationship between the clinical outcome and specific resistance genes are limited. A previous study showed that mortality in critical patients with A. baumannii complex bacteremia was not correlated with any specific genospecies (Lee et al., 2014). Resistance mechanisms might not play a significant role in the clinical outcomes of patients with CRAB infection, but further studies are needed.

For strains with the presence of carbapenemases, more isolates exhibited synergistic activity with a combination of PB/TGC and SUL, but there was no statistically significant difference in the synergistic effect for the strains with overexpression of efflux pump genes and functional loss of porin. Leelasupasri et al. (2018) also found that colistin combined with SUL has a good synergistic and additive effect on CRAB strains, and colistin plus SUL against CRAB seemed to be an interesting option. There are limited studies on the in vitro antibacterial activity of an antimicrobial combination based on different resistance mechanisms. An open-label, randomized controlled trial found that colistin-meropenem combination was not superior to colistin monotherapy in the cumulative survival rate (Paul et al., 2018). Although many combinations, such as SUL-TGC, SUL-carbapenems, polymyxin-carbapenems, and polymyxin-TGC, were used for CRAB infection (Chinese XDR Consensus Working Group et al., 2016), the efficacy of colistinSUL against CRAB in clinical use also has to be evaluated. The 28day treatment failure rate in these patients was 28.05\% (23/82). The patients at an advanced age, those with MODS, those with a lower level of eGFR, and those admitted to the ICU had a poor clinical outcome, which was similar to that in previous studies (Sheng et al., 2010; Niu et al., 2018; Du et al., 2019). The treatment failure rate increased with the advanced age of patients, possibly associated with a decreased immune response to pathogens as age increases (Li M. et al., 2019). MODS, a lower level of eGFR, and ICU stay often indicate that the patients are critically ill and are more likely to die.

This study also showed that the level of PCT in the treatment failure group was significantly higher than that in the treatment success group. PCT has now been considered as a marker for early diagnosis, prognosis, and antibiotic stewardship in patients with bacterial infections, especially acute respiratory infections and sepsis (Lee et al., 2020).

Some previous studies have shown that appropriate therapy could decrease the mortality in patients with CRE bloodstream infections (Gutiérrez-Gutiérrez et al., 2017; Wang et al., 2018). In this study, we found that appropriate antibiotic therapy did not improve the clinical outcome of patients with CRAB infection. Most of the patients in the treatment failure group were in a critical condition when they developed CRAB infection. MODS is associated with very high mortality (Ramírez, 2013), and their clinical outcome was still poor even if they were given aggressive antibiotic therapy. Therefore, early identification of bacterial infection, immediate administration of antibiotic therapy, and prevention of severe infection-induced MODS are very important to reduce bacterial infection-related mortality. This study also found that the group that died had higher TGC MICs. Some patients were given TGC because polymyxin was not available in China at that time. Lower MICs of TGC 
can more easily meet the pharmacokinetic/pharmacodynamic parameter requirements.

In our study, multivariate analysis showed that ICU stay and PCT $\geq 2 \mathrm{ng} / \mathrm{ml}$ were closely correlated with a poor outcome of patients with CRAB infection. Previous studies showed that there were many risk factors for treatment failure in CRAB infection, including ICU stay, TGC therapy, presence of septic shock, higher acute Physiology and Chronic Health Evaluation II (APACHE II) score, central venous catheterization, inappropriate empirical antimicrobial treatment, and multiple organ failure (Sheng et al., 2010; Niu et al., 2018; Du et al., 2019). High PCT values often represent severe infection, which often occurs in critically ill patients and easily leads to death. A previous systematic review found that inappropriate empirical antimicrobial treatment was a risk factor for higher mortality in patients with CRAB infections (Du et al., 2019), which was different from that in our study. Further multicenter, highquality, prospective studies are needed.

This study has some limitations. First, since the MIC values of TGC and polymyxin of many strains were very low, only some strains were evaluated for the in vitro antibacterial activity of an antimicrobial combination. Second, only three patients were treated with polymyxin because it was not available in our hospital at that time. Therefore, clinical data on polymyxin combined with SUL was not available. Relevant studies will be carried out in the future.

\section{CONCLUSION}

In conclusion, this study demonstrated the synergistic potential of the combination of polymyxin/TGC and SUL in vitro against CRAB presenting carbapenemase-encoding genes. The clinical outcome of patients was not associated with resistance mechanisms. The patients at an advanced age, those with MODS, and those admitted to the ICU had a poor clinical outcome. ICU stay and PCT $\geq 2 \mathrm{ng} / \mathrm{ml}$ were risk factors for treatment

\section{REFERENCES}

Bae, S., Kim, M. C., Park, S. J., Kim, H. S., Sung, H., Kim, M. N., et al. (2016). In Vitro synergistic activity of antimicrobial agents in combination against clinical isolates of colistin-resistant Acinetobacter baumannii. Antimicrob. Agents. Chemother. 60, 6774-6779. doi: 10.1128/AAC.00839-16

Brink, A. J. (2019). Epidemiology of carbapenem-resistant Gram-negative infections globally. Curr. Opin. Infect. Dis. 32, 609-616. doi: 10.1097/qco. 0000000000000608

Chinese XDR Consensus Working Group, Guan, X., He, L., Hu, B., Hu, J., Huang, X., et al. (2016). Laboratory diagnosis, clinical management and infection control of the infections caused by extensively drug-resistant Gram-negative bacilli: a Chinese consensus statement. Clin. Microbiol. Infect. 22(Suppl. 1), S15-S25. doi: 10.1016/j.cmi.2015.11.004

CHINET (2019). Institute of Antibiotics, Huashan Hospital, Fudan University. Available online at: http://www.chinets.com/Data/GermYear (accessed January $5,2020)$.

CLSI (2017). Performance Standards for Antimicrobial Susceptibility Testing. 27th ed. CLSI Supplement M100. Wayne, PA: Clinical and Laboratory Standard Institute.

Doi, Y. (2019). Treatment options for carbapenem-resistant gram-negative bacterial infections. Clin. Infect. Dis. 69, S565-S575. doi: 10.1093/cid/ciz830 failure in patients with $\mathrm{CRAB}$ infection. Appropriate antibiotic therapy did not improve the clinical outcome of critically ill patients. Infections with higher MICs of TGC were more difficult to control. It may be more important to identify the infection early, actively control its progression to severe infection, and reduce ICU admission.

\section{DATA AVAILABILITY STATEMENT}

All datasets generated for this study are included in the article/supplementary material.

\section{ETHICS STATEMENT}

The studies involving human participants were reviewed and approved by the Ethics Committee of West China Hospital, Sichuan University. Written informed consent for participation was not required for this study in accordance with the national legislation and the institutional requirements.

\section{AUTHOR CONTRIBUTIONS}

XL and JQ conceived of and designed the study. XL supervised the experiments. JQ, RY, and CF performed the experiments. QW and CF collected the data. JQ, RY, and QW analyzed or interpreted the data. JQ wrote the draft. All authors read, revised, and approved the final manuscript.

\section{FUNDING}

This study was supported by the Sichuan Province Science and Technology Support Program of China (Grant Number: 2017SZ0140).

Du, X., Xu, X., Yao, J., Deng, K., Chen, S., Shen, Z., et al. (2019). Predictors of mortality in patients infected with carbapenem-resistant Acinetobacter baumannii: a systematic review and meta-analysis. Am. J. Infect. Control 47, 1140-1145. doi: 10.1016/j.ajic.2019.03.003

Ellington, M. J., Kistler, J., Livermore, D. M., and Woodford, N. (2007). Multiplex PCR for rapid detection of genes encoding acquired metallo-beta-lactamases. J. Antimicrob. Chemother. 59, 321-322. doi: 10.1093/jac/dkl481

Elsayed, E., Elarabi, M. A., Sherif, D. A., Elmorshedi, M., and El-Mashad, N. (2019). Extensive drug resistant Acinetobacter baumannii: a comparative study between non-colistin based combinations. Int. J. Clin. Pharm. 42, 80-88. doi: 10.1007/s11096-019-00940-1

Goh, H. M., Beatson, S. A., Totsika, M., Moriel, D. G., Phan, M. D., Szubert, J., et al. (2013). Molecular analysis of the Acinetobacter baumannii biofilm-associated protein. Appl. Environ. Microbiol. 79, 6535-6543. doi: 10.1128/AEM.01402-13

Gordon, N. C., Png, K., and Wareham, D. W. (2010). Potent synergy and sustained bactericidal activity of a vancomycin-colistin combination versus multidrugresistant strains of Acinetobacter baumannii. Antimicrob. Agents Chemother. 54, 5316-5322. doi: 10.1128/aac.00922-10

Gutiérrez-Gutiérrez, B., Salamanca, E., de Cueto, M., Hsueh, P. R., Viale, P., Paño-Pardo, J. R., et al. (2017). Effect of appropriate combination therapy on mortality of patients with bloodstream infections due to carbapenemase-producing Enterobacteriaceae (INCREMENT): a retrospective 
cohort study. Lancet Infect. Dis. 17, 726-734. doi: 10.1016/S1473-3099(17) 30228-1

Haidar, G., Clancy, C. J., Shields, R. K., Hao, B., Cheng, S., and Nguyen, M. H. (2017). Mutations in blaKPC-3 that confer ceftazidime-avibactam resistance encode novel KPC-3 variants that function as extended-spectrum $\beta$ Lactamases. Antimicrob. Agents Chemother. 61:e2534-16. doi: 10.1128/AAC. 02534- 16

He, S., He, H., Chen, Y., Chen, Y., Wang, W., and Yu, D. (2015). In vitro and in vivo analysis of antimicrobial agents alone and in combination against multi-drug resistant Acinetobacter baumannii. Front. Microbiol. 6:507. doi: 10.3389/fmicb. 2015.00507

Higgins, P. G., Lehmann, M., and Seifert, H. (2010). Inclusion of OXA-143 primers in a multiplex polymerase chain reaction (PCR) for genesencoding prevalent OXA carbapenemases in Acinetobacter spp. Int. J. Antimicrob. Agents 35:305. doi: 10.1016/j.ijantimicag.2009.10.014

Horan, T. C., Andrus, M., and Dudeck, M. A. (2008). CDC/NHSN surveillance definition of healthcare-associated infection and criteria for specific types of infections in the acute care setting. Am. J. Infect. Control 36, 309-332. doi: 10.1016/j.ajic.2008.03.002

Hsueh, S. C., Lee, Y. J., Huang, Y. T., Liao, C. H., Tsuji, M., and Hsueh, P. R. (2019), In vitro activities of cefiderocol, ceftolozane/tazobactam, ceftazidime/avibactam and other comparative drugs against imipenem-resistant Pseudomonas aeruginosa and Acinetobacter baumannii, and Stenotrophomonas maltophilia, all associated with bloodstream infections in Taiwan. J. Antimicrob. Chemother. 74, 380-386. doi: 10.1093/jac/dky425

Jiang, L., Liang, Y., Yao, W., Ai, J., Wang, X., and Zhao, Z. (2019). Molecular epidemiology and genetic characterisation of carbapenem-resistant Acinetobacter baumannii isolates from Guangdong Province. South China. J. Glob. Antimicrob. Resist. 17, 84-89. doi: 10.1016/j.jgar.2018.11.002

Karaiskos, I., Antoniadou, A., and Giamarellou, H. (2017). Combination therapy for extensively-drug resistant gram-negative bacteria. Expert. Rev. Anti. Infect. Ther. 15, 1123-1140. doi: 10.1080/14787210.2017.1410434

Kim, S. Y., Jung, J. Y., Kang, Y. A., Lim, J. E., Kim, E. Y., Lee, S. K., et al. (2012). Risk factors for occurrence and 30-day mortality for carbapenem-resistant Acinetobacter baumannii bacteremia in an intensive care unit. J. Korean Med. Sci. 27, 939-947. doi: 10.3346/jkms.2012.27.8.939

Le Minh, V., Thi Khanh Nhu, N., Vinh Phat, V., Thompson, C., Huong Lan, N. P., Thieu Nga, T. V., et al. (2015). In vitro activity of colistin in antimicrobial combination against carbapenem-resistant Acinetobacter baumannii isolated from patients with ventilator-associated pneumonia in Vietnam. J. Med. Microbiol. 64, 1162-1169. doi: 10.1099/jmm.0.000137

Lee, C. C., Kwa, A. L. H., Apisarnthanarak, A., Feng, J. Y., Gluck, E. H., Ito, A., et al. (2020). Procalcitonin (PCT)-guided antibiotic stewardship in Asia-Pacific countries: adaptation based on an expert consensus meeting. Clin. Chem. Lab. Med. doi: 10.1515/cclm-2019-1122 [Epub ahead of print].

Lee, H. Y., Chen, C. L., Wu, S. R., Huang, C. W., and Chiu, C. H. (2014). Risk factors and outcome analysis of Acinetobacter baumannii complex bacteremia in critical patients. Crit. Care Med. 42, 1081-1088. doi: 10.1097/ ccm.0000000000000125

Leelasupasri, S., Santimaleeworagun, W., and Jitwasinkul, T. (2018). Antimicrobial susceptibility among colistin, sulbactam, and fosfomycin and a synergism study of colistin in combination with sulbactam or fosfomycin against clinical isolates of carbapenem-resistant Acinetobacter baumannii. J. Pathog. 2018:3893492. doi: 10.1155/2018/3893492

Leone, S., Damiani, G., Pezone, I., Kelly, M. E., Cascella, M., Alfieri, A., et al. (2019). New antimicrobial options for the management of complicated intraabdominal infections. Eur. J. Clin. Microbiol. Infect. Dis. 38, 819-827. doi: 10.1007/s10096-019-03533-y

Li, M., Yao, D., Zeng, X., Kasakovski, D., Zhang, Y., Chen, S., et al. (2019). Age related human T cell subset evolution and senescence. Immun. Ageing 16:24. doi: 10.1186/s12979-019-0165-8

Li, S., Duan, X., Peng, Y., and Rui, Y. (2019). Molecular characteristics of carbapenem-resistant Acinetobacter spp. from clinical infectionsamples and fecal survey samples in Southern China. BMC Infect. Dis. 19:900. doi: 10.1186/ s12879-019-4423-3

Livak, K. J., and Schmittgen, T. D. (2001). Analysis of relative gene expression data using real-time quantitative PCR and the 2(-Delta Delta C(T)) Method. Methods 25, 402-408. doi: 10.1006/meth.2001.1262
McGovern, P. C., Wible, M., El-Tahtawy, A., Biswas, P., and Meyer, R. D. (2013). All-cause mortality imbalance in the tigecycline phase 3 and 4 clinical trials. Int. J. Antimicrob. 41, 463-467. doi: 10.1016/j.ijantimicag.2013. 01.020

Niu, T., Xiao, T., Guo, L., Yu, W., Chen, Y., Zheng, B., et al. (2018). Retrospective comparative analysis of risk factors and outcomes in patients with carbapenem-resistant Acinetobacter baumannii bloodstream infections: cefoperazone-sulbactam associated with resistance and tigecycline increased the mortality. Infect. Drug. Resist. 11, 2021-2030. doi: 10.2147/idr.s16 9432

Paul, M., Daikos, G. L., Durante-Mangoni, E., Yahav, D., Carmeli, Y., Benattar, Y. D., et al. (2018). Colistin alone versus colistin plus meropenem for treatment of severe infections caused by carbapenem-resistant Gram-negative bacteria: an open-label, randomised controlled trial. Lancet Infect. Dis. 18, 391-400. doi: 10.1016/s1473-3099(18)30099-9

Peleg, A. Y., Adams, J., and Paterson, D. L. (2007). Tigecycline efflux as a mechanism for nonsusceptibility in Acinetobacter baumannii. Antimicrob. Agents Chemother. 51, 2065-2069. doi: 10.1128/aac.01198-06

Poirel, L., Le Thomas, I., Naas, T., Karim, A., and Nordmann, P. (2000). Biochemical sequence analyses of GES-1, a novel class A extended-spectrum beta-lactamase, and the class 1 integron In52 from Klebsiella pneumoniae. Antimicrob. Agents Chemother. 44, 622-632. doi: 10.1128/aac.44.3.622-632. 2000

Ramírez, M. (2013). Multiple organ dysfunction syndrome. Curr. Probl. Pediatr. Adolesc. Health Care 43, 273-277. doi: 10.1016/j.cppeds.2013.10.003

Russo, A., Bassetti, M., Ceccarelli, G., Carannante, N., Losito, A. R., Bartoletti, M., et al. (2019). Bloodstream infections caused by carbapenem-resistant Acinetobacter baumannii: clinical features, therapy and outcome from a multicenter study. J. Infect. 79, 130-138. doi: 10.1016/j.jinf.2019.05.017

Sheng, W. H., Liao, C. H., Lauderdale, T. L., Ko, W. C., Chen, Y. S., Liu, J. W., et al. (2010). A multicenter study of risk factors and outcome of hospitalized patients with infections due to carbapenem-resistant Acinetobacter baumannii. Int. J. Infect. Dis. 14:e764-e9. doi: 10.1016/j.ijid.2010.02.2254

Wang, X., Wang, Q., Cao, B., Sun, S., Zhang, Y., Gu, B., et al. (2018). Retrospective observational study from a chinese network of the impact of combination therapy versus monotherapy on mortality from carbapenemresistant Enterobacteriaceae bacteremia. Antimicrob. Agents Chemother. 63:e1511-18. doi: 10.1128/AAC.01511-18

Willyard, C. (2017). The drug-resistant bacteria that pose the greatest health threats. Nature 543:15. doi: 10.1038/nature.2017.21550

Woodford, N., Ellington, M. J., Coelho, J. M., Turton, J. F., Ward, M. E., Brown, S., et al. (2006). Multiplex PCR for genes encoding prevalent OXA carbapenemases in Acinetobacter spp. Int. J. Antimicrob. Agents 27, 351-353. doi: 10.1016/j. ijantimicag.2006.01.004

Yazdansetad, S., Najari, E., Ghaemi, E. A., Javid, N., Hashemi, A., and Ardebili, A. (2019). Carbapenem-resistant Acinetobacter baumannii isolates carrying blaOXA genes with upstream ISAbal: first report of a novel OXA subclass from Iran. J. Glob. Antimicrob. Resist. 18, 95-99. doi: 10.1016/j.jgar.2018. 12.011

Yoon, J., Urban, C., Terzian, C., Mariano, N., and Rahal, J. J. (2004). In vitro double and triple synergistic activities of Polymyxin B, imipenem, and rifampin against multidrug-resistant Acinetobacter baumannii. Antimicrob. Agents Chemother. 48, 753-757. doi: 10.1128/aac.48.3.753-757.2004

Zusman, O., Altunin, S., Koppel, F., Dishon Benattar, Y., Gedik, H., and Paul, M. (2017). Polymyxin monotherapy or in combination against carbapenemresistant bacteria: systematic review and meta-analysis. J. Antimicrob. Chemother. 72, 29-39. doi: 10.1093/jac/dkw377

Conflict of Interest: The authors declare that the research was conducted in the absence of any commercial or financial relationships that could be construed as a potential conflict of interest.

Copyright (c) $2020 \mathrm{Qu}, \mathrm{Yu}$, Wang, Feng and Lv. This is an open-access article distributed under the terms of the Creative Commons Attribution License (CC BY). The use, distribution or reproduction in other forums is permitted, provided the original author(s) and the copyright owner(s) are credited and that the original publication in this journal is cited, in accordance with accepted academic practice. No use, distribution or reproduction is permitted which does not comply with these terms. 